Supporting Information for

\title{
Pre-formed biodegradable zwitterionic nanoparticles as tunable excipients for the formulation of therapeutics directly at the point of care
}

\author{
Renato Auriemma ${ }^{1}$, Mattia Sponchioni ${ }^{1 *}$, Sophia Lotti ${ }^{1}$, Lavinia Morosi ${ }^{2}$, Massimo Zucchetti ${ }^{2}$, Monica Lupi ${ }^{2}$, \\ Davide Moscatelli ${ }^{1}$, Umberto Capasso Palmiero ${ }^{3 *}$
}

${ }^{1}$ Department of Chemistry, Materials and Chemical Engineering, Politecnico di Milano, Via Mancinelli 7 - 20131 Milano, Italy.

${ }^{2}$ Istituto di Ricerche Farmacologiche Mario Negri IRCCS, Department of Oncology, 20156 Milano, Italy.

${ }^{3}$ Department of Chemistry and Applied Biosciences, Institute for Chemical and Bioengineering, ETH Zurich, Switzerland.

* corresponding authors: Mattia Sponchioni, e-mail: mattia.sponchioni@polimi.it Umberto Capasso Palmiero, e-mail: umberto.capasso@chem.ethz.ch 
Synthesis of the Macromolecular Chain Transfer Agent (Macro CTA)

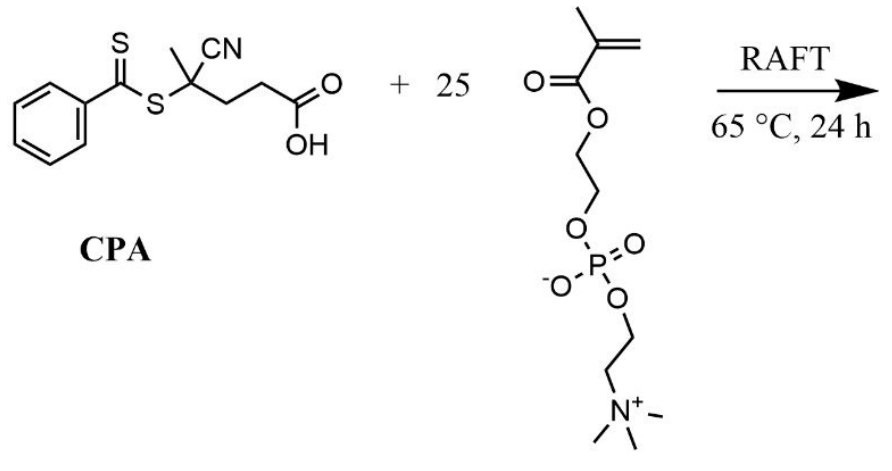

MPC

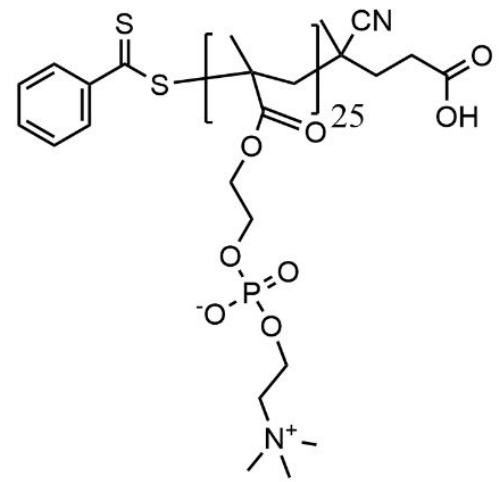

25MPC

\section{Macromonomer Synthesis}

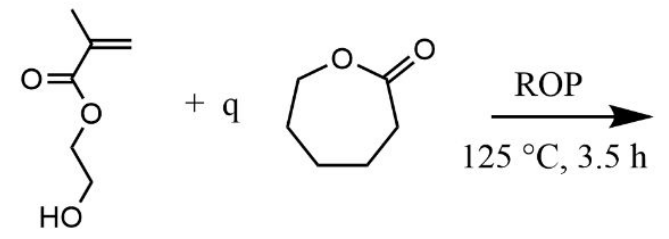

HEMA

$\varepsilon$-caprolactone<smiles>C=C(C)C(=O)OCCOC(=O)CCCCCO</smiles>

HEMA-CLq

\section{Neutral NPs}

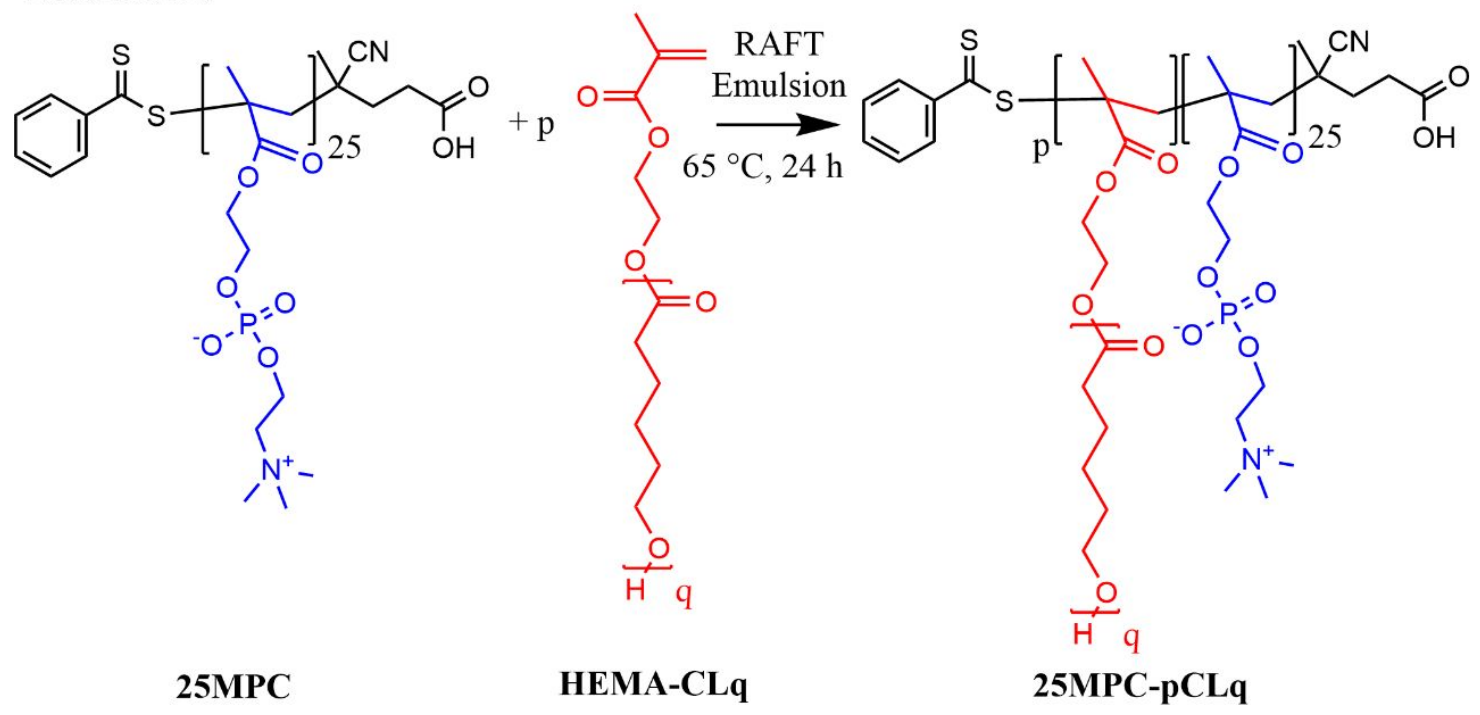

Scheme S1: Synthesis of the zwitterionic macromolecular chain transfer agent 25MPC via RAFT solution polymerization, of the macromonomer HEMA-CLq and of the amphiphilic block copolymers 25MPC-pCLq self-assembled into neutral NPs 


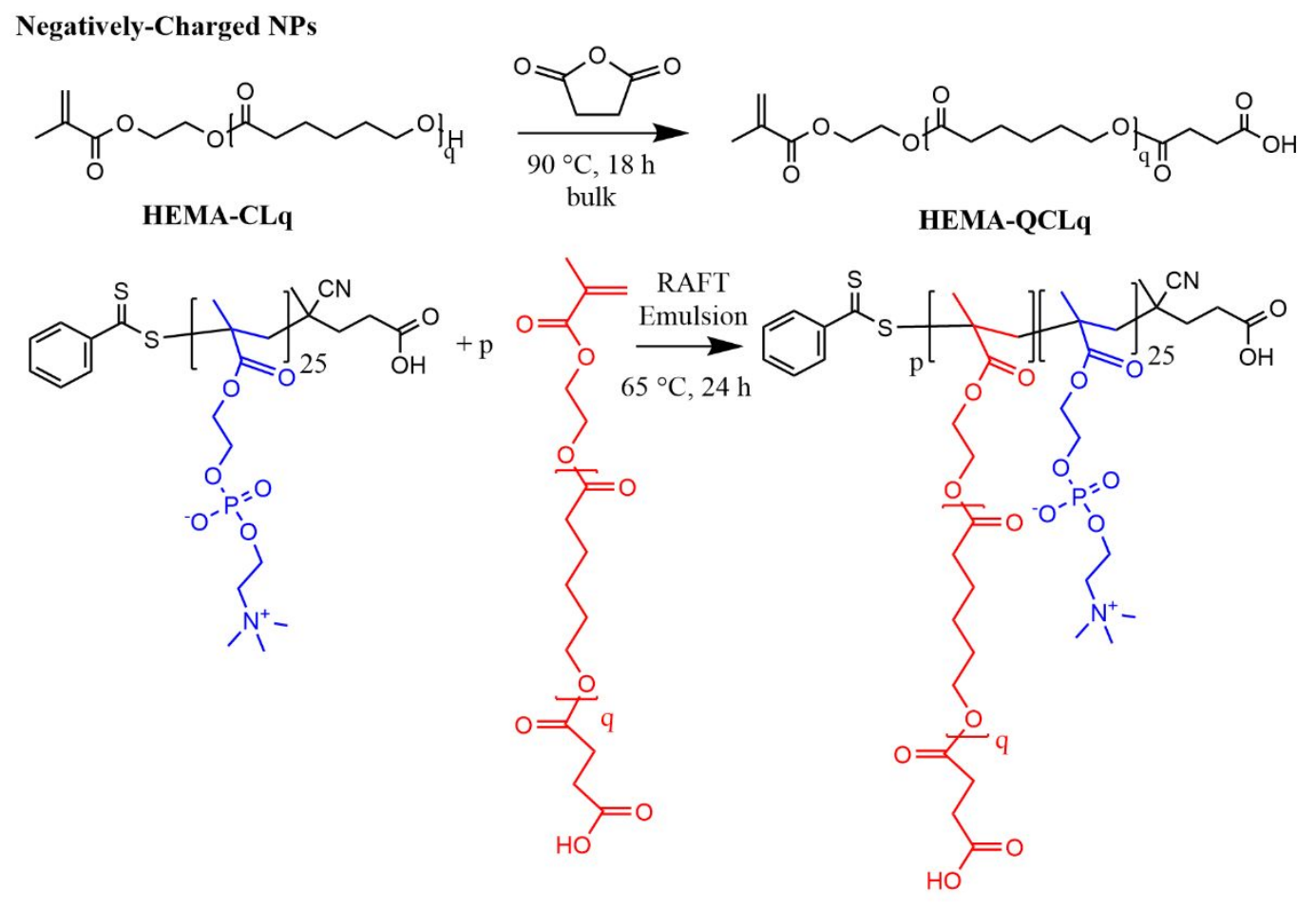

25MPC

HEMA-QCLq

25MPC-pQCLq
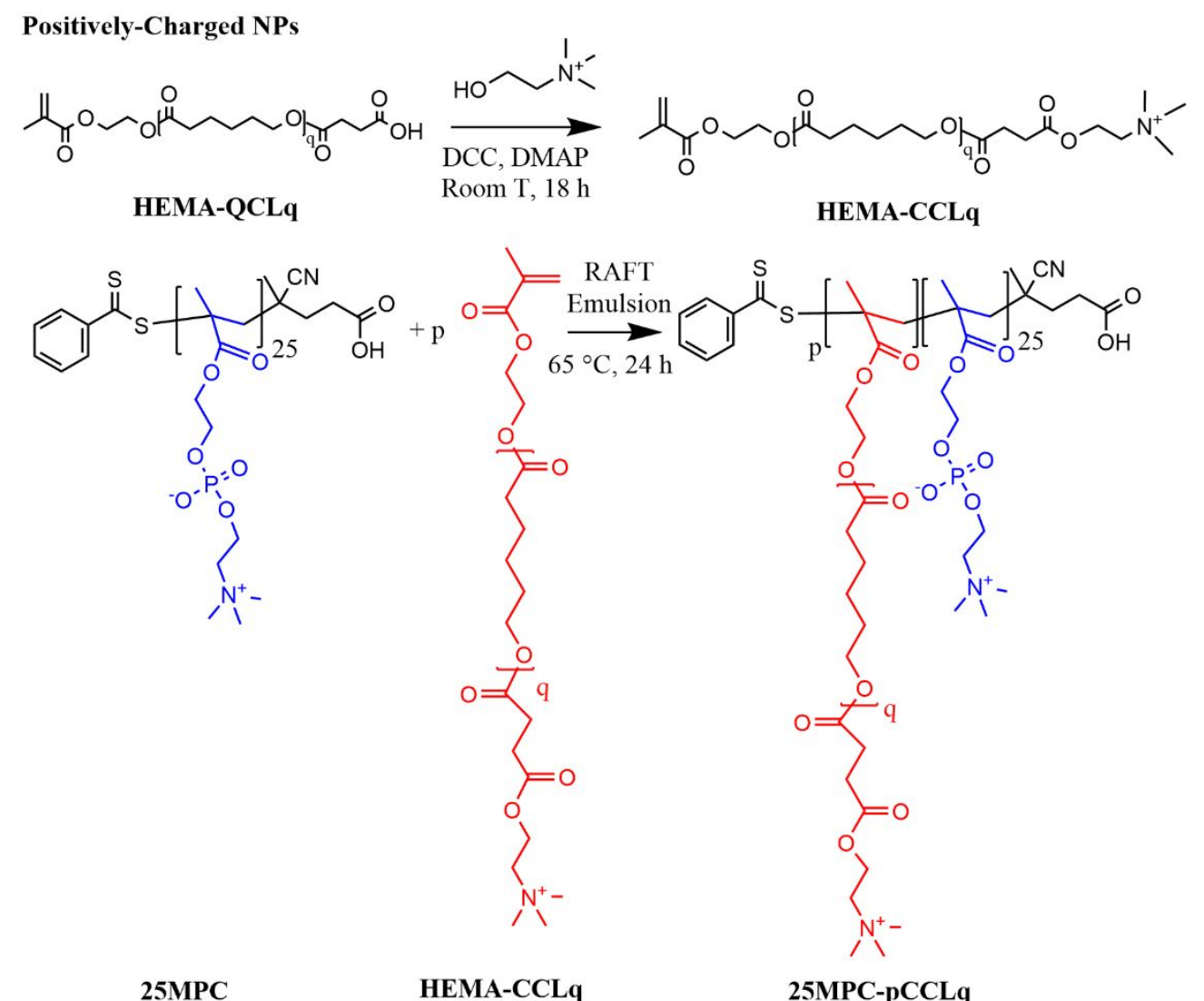

Scheme S2: Functionalization of the macromonomer to obtain negatively-charged NPs (i.e. 25MPC-pQCLq) and positively-charged NPs (i.e. 25MPC-pCCLq) 

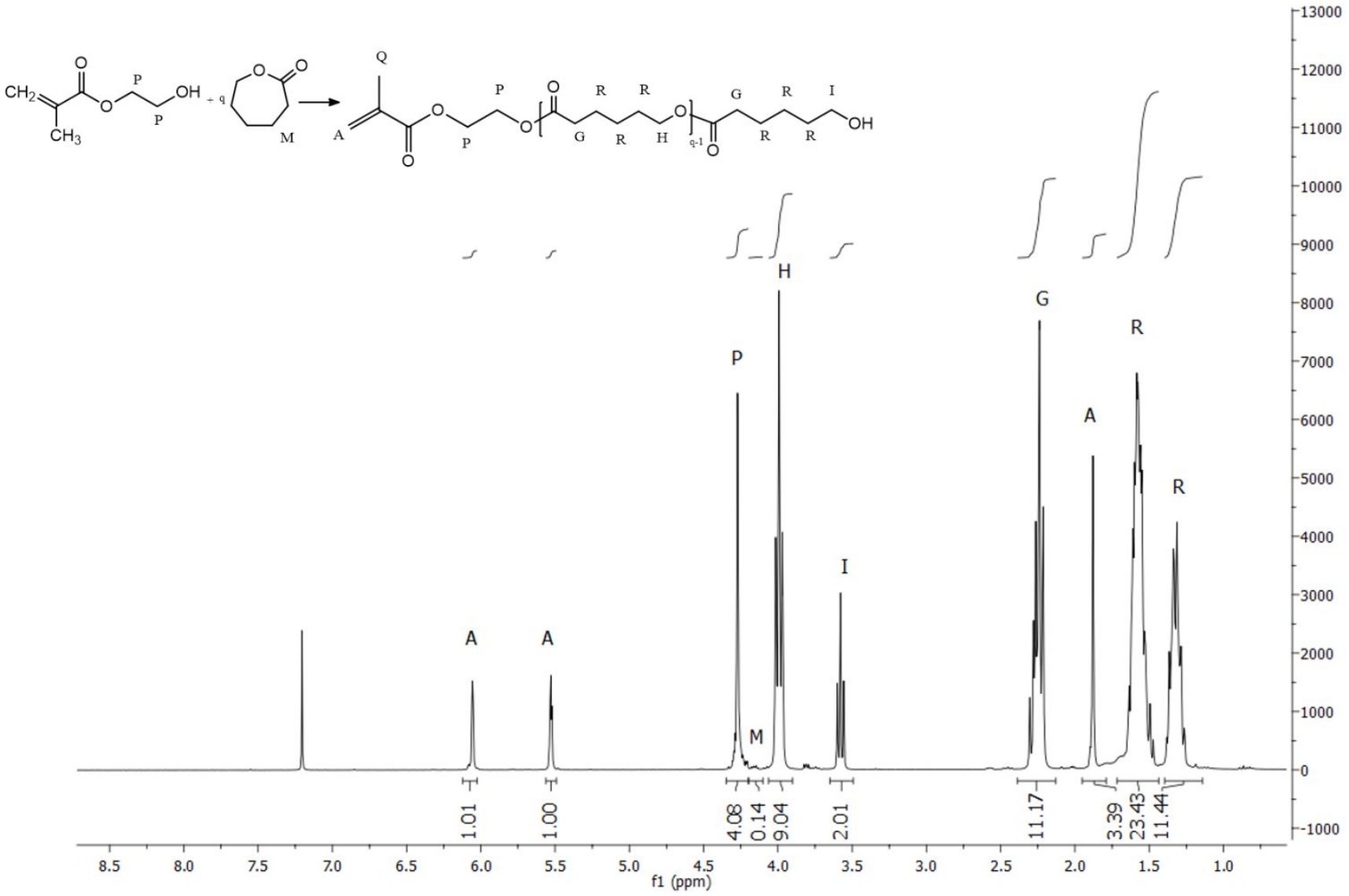

Figure S1: Representative ${ }^{1} \mathrm{H}-\mathrm{NMR}$ spectrum of $\mathrm{HEMA}-\mathrm{CL}_{\mathrm{q}}$ macromonomer with $\mathrm{q}=5$

The DP $(q)$ of HEMA-CLq is calculated as described in eq. (S1):

$q=\frac{2(H+I)}{P}$

The peaks I and $\mathrm{H}$, represent respectively the two hydrogen atoms adjacent to the chain-end hydroxyl group and to the ester group, while P refers to the 4 protons of the HEMA used as initiator.

The conversion $X_{C L}$, was calculated according to eq. (S2):

$X_{C L}=\frac{H+I}{H+I+M}$ 
The peak $\mathrm{M}$ of the two hydrogens adjacent to the ester group of the monomeric CL was compared to the peaks $\mathrm{H}$ and I, of the two hydrogen atoms adjacent to the chain-end hydroxyl group and to the ester group of the polymerized CL, respectively.

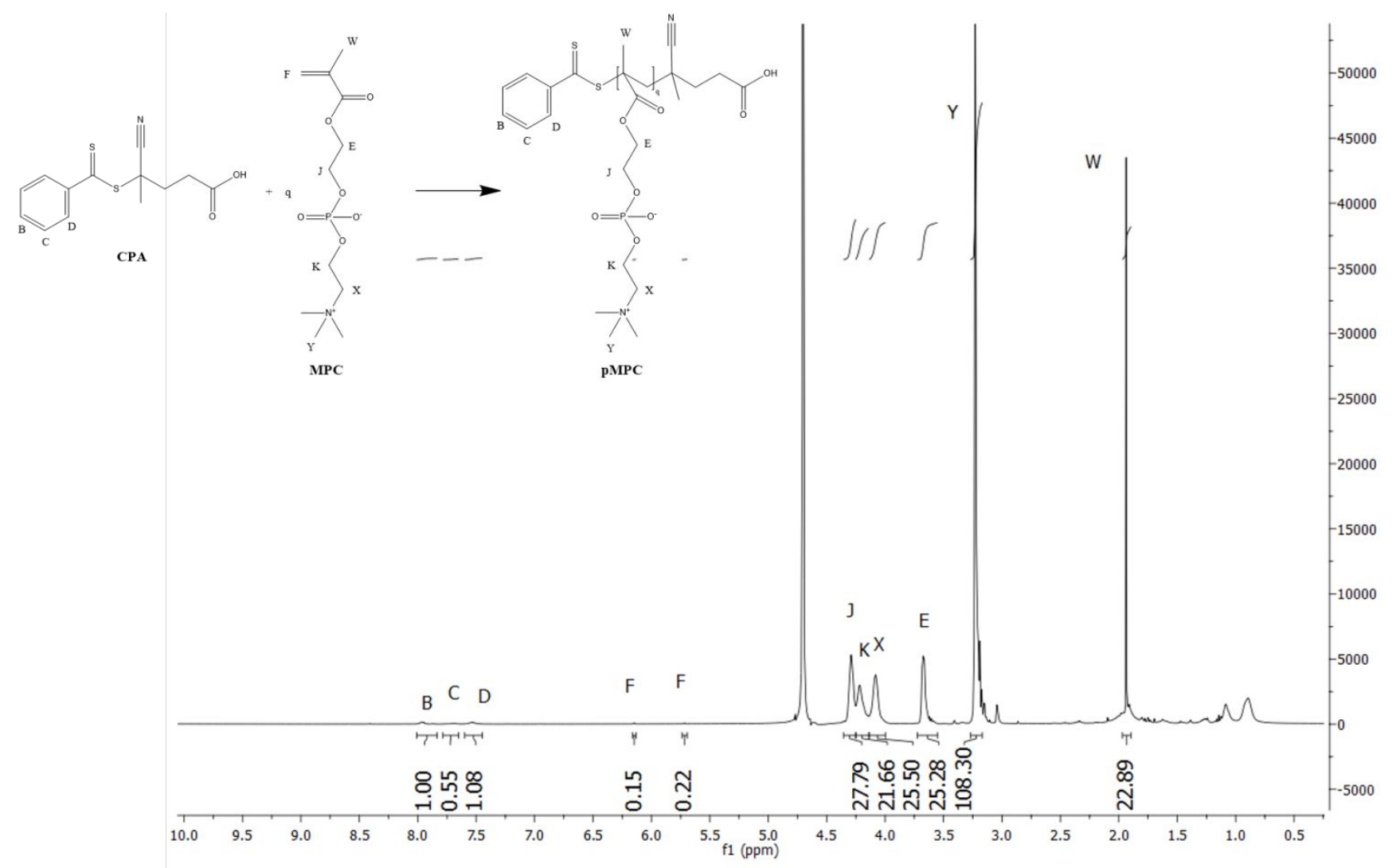

Figure S2: Representative ${ }^{1} \mathrm{H}-\mathrm{NMR}$ spectrum of the nMPC macromolecular RAFT agent with $\mathrm{n}=25$

The DP $(n)$ of poly-MPC can be calculated as described in eq. (S3):

$n_{M P C}=\frac{E}{D} * X_{M P C}$ 
The peak D that belongs to CPA, was chosen as reference and its area was set equal to one. To compute the DP, peak E was employed as it belongs to the two hydrogen atoms of the methylene group of both the monomeric and polymerized MPC.

The conversion $X_{M P C}$ instead is calculated according to eq. (S4):

$X_{M P C}=1-\frac{2 * F}{E}$

To calculate the monomer conversion, the peak of one of the hydrogen atoms of the double bond (F), only present on the unreacted monomer, was compared to the peak E correspondent to two hydrogen atoms, referred to the converted polymer and unreacted monomer. 


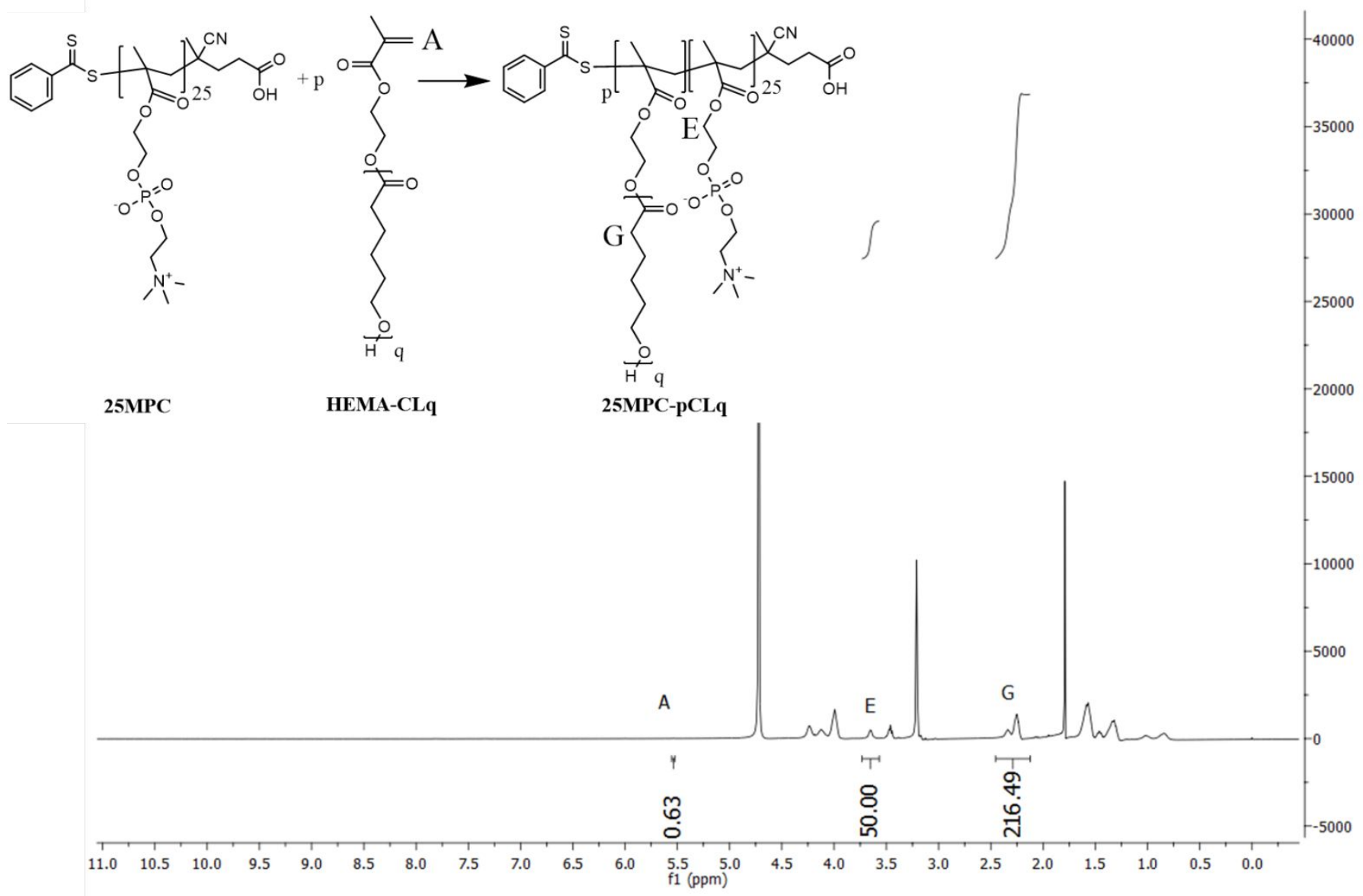

Figure S3: Representative ${ }^{1} \mathrm{H}-\mathrm{NMR}$ spectrum of the 25MPC-pCLq-based nanoparticles with $\mathrm{q}=5$ and $\mathrm{p}=30$

The peak E correspondent to the two hydrogen atoms present only in MPC was chosen as a reference and set equal to 50 ( 2 hydrogen atoms of the methylene group multiplied by 25 units of MPC).

The DP $(p)$ can be calculated as described in eq. (S5):

$p=\frac{n M P C * G}{q * E}$

The peak $\mathrm{G}$ refers to the two hydrogen atoms present in both reacted and unreacted macro-monomer units.

The conversion $X_{H E M A-C L q / M P C}$ is determined according to eq. (S6):

$X_{H E M A C L q / M P C}=1-\frac{2 * q * A}{G}$ 
The peak A, correspondent to one of the two hydrogen atoms of the lipophilic monomer double bond and the peak $\mathrm{G}$ were weighted on their number of hydrogens, divided and the final product was subtracted from 1 .

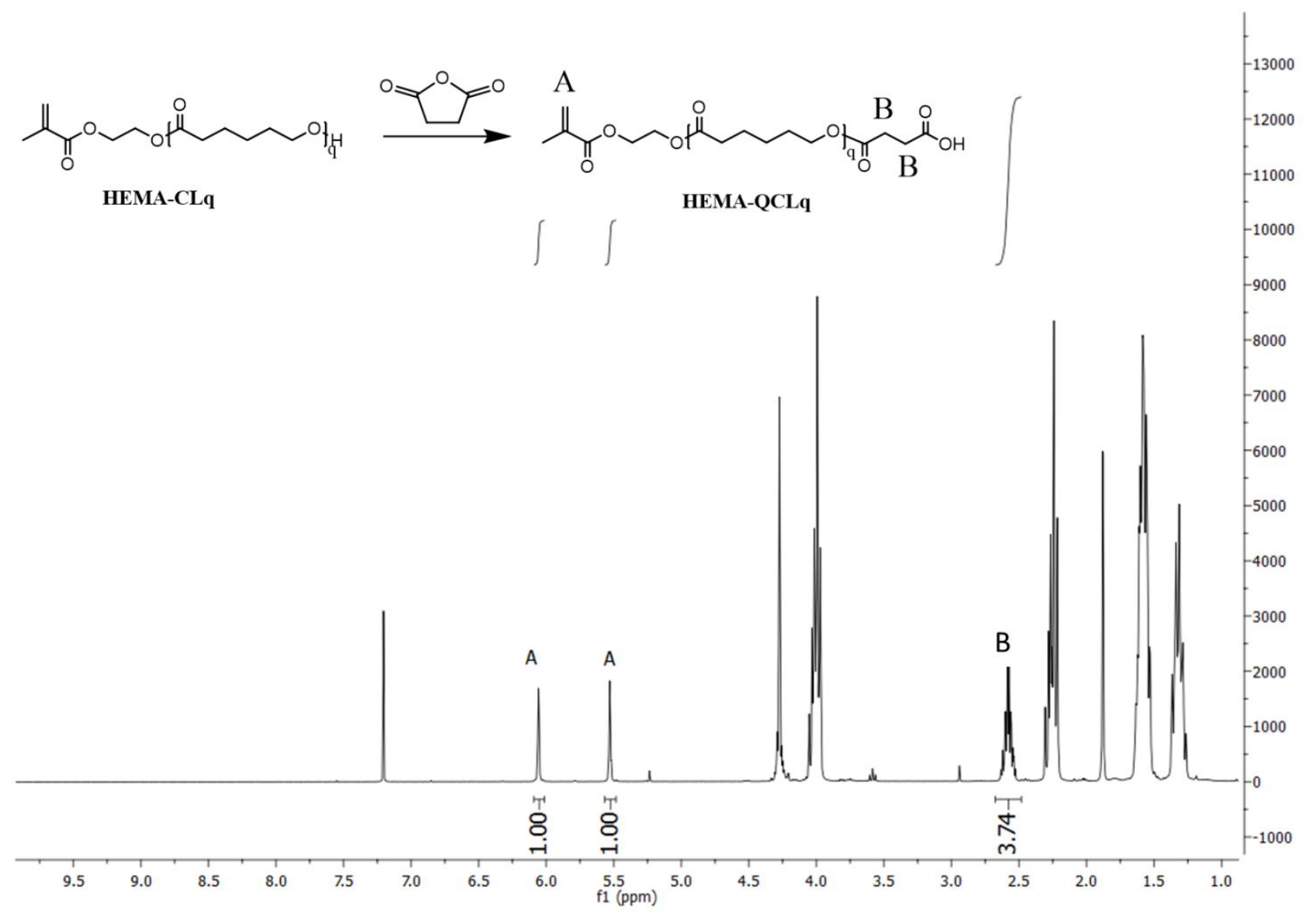

Figure S4: Representative ${ }^{1} \mathrm{H}-\mathrm{NMR}$ spectrum of $\mathrm{HEMA}-\mathrm{QCL}_{\mathrm{q}}$ macromonomer with $\mathrm{q}=5$ synthesized via succynilation of HEMA-CL 5

One of the hydrogen atoms of the double bond present in HEMA-CLq (A) has been chosen as reference and its area has been set equal to one.

The functionalization with a carboxyl group to the chain and the disappearance of the hydroxyl group has caused the appearance of the peak B corresponding to the four hydrogen atoms adjacent to the carboxylic group. As a consequence, the disappearance of the peak I (Figure S1) regarding the two hydrogen atoms adjacent to the chain-end hydroxyl group has occurred causing an increase in the area of the peak $\mathrm{H}$ that refers to the two hydrogen atoms near the ester group. 


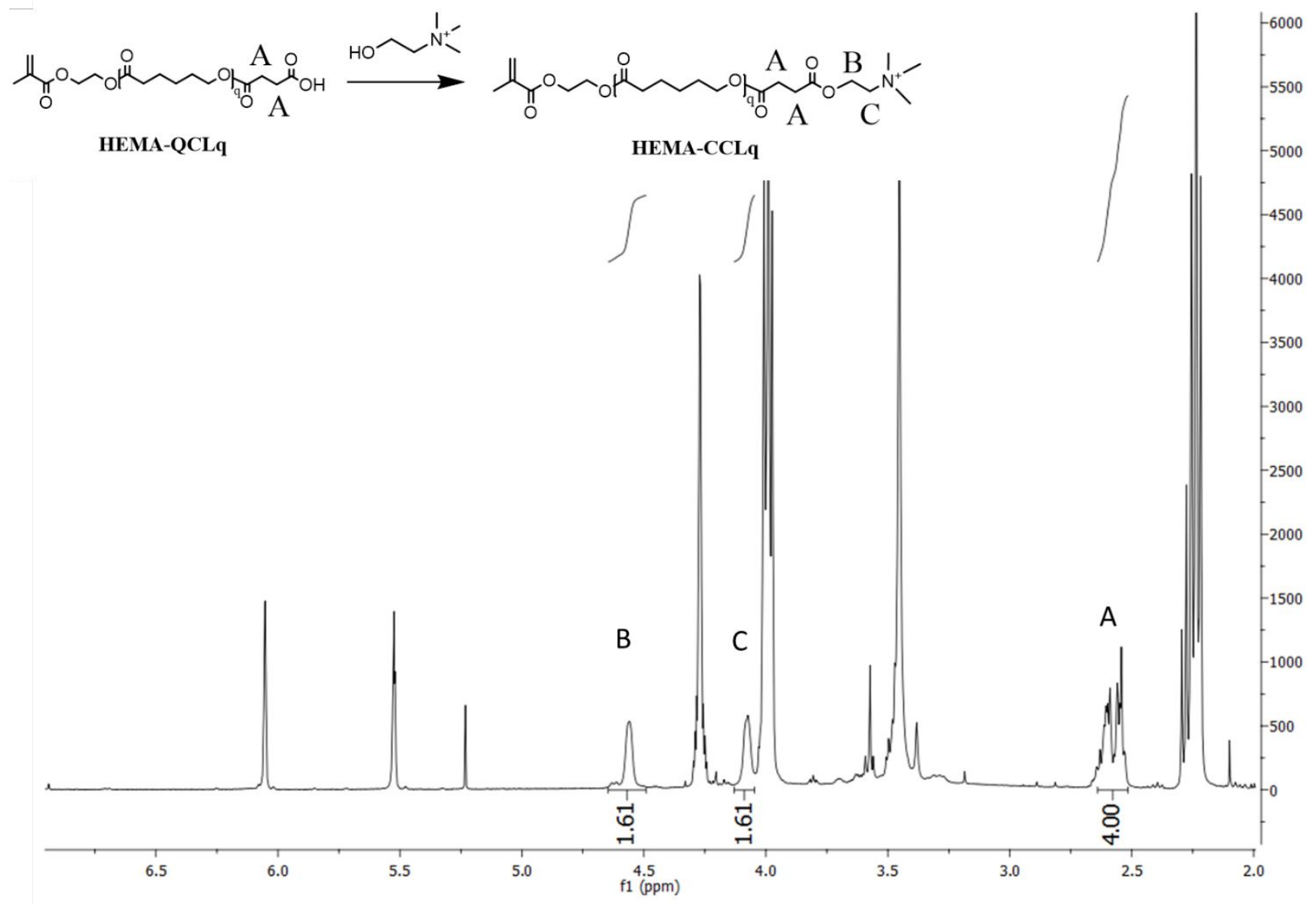

Figure S5: Representative ${ }^{1} \mathrm{H}-\mathrm{NMR}$ spectrum of HEMA-CCL $\mathrm{q}_{\mathrm{q}}$ macromonomer with $\mathrm{q}=5$ synthesized via DCC-mediated esterification of HEMA-QCL 5 with choline chloride

The conversion $X_{C C L}$, was calculated according to eq. (S7):

$X_{C C L}=\frac{B+C}{A}$ 


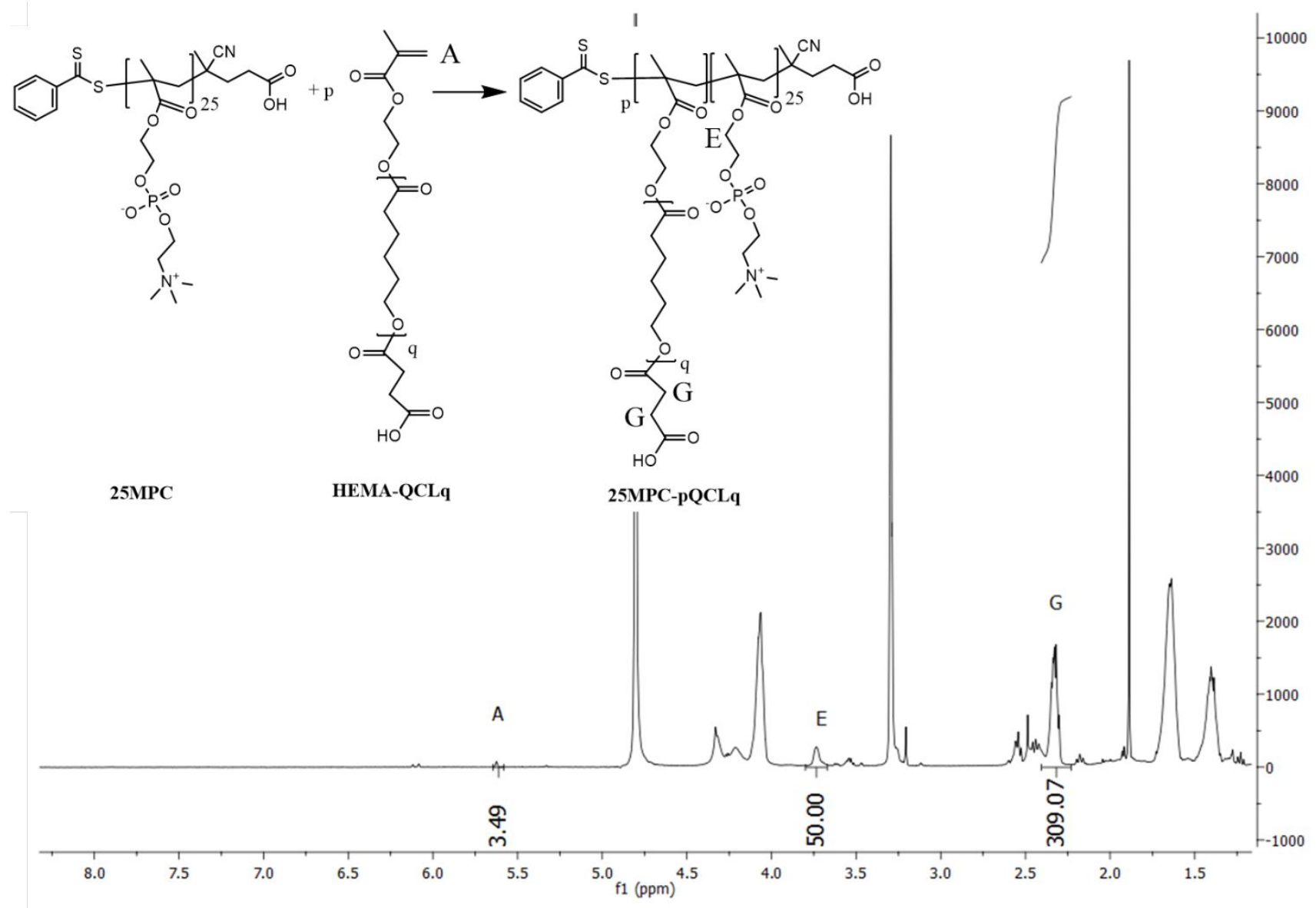

Figure S6: Representative ${ }^{1} \mathrm{H}-\mathrm{NMR}$ spectrum of the $25 \mathrm{MPC}-\mathrm{pQCLq}$-based nanoparticles with $\mathrm{q}=5$ and $\mathrm{p}=30$

Analyzing this spectrum, it was possible to calculate the conversion with respect to the macromonomer HEMA-QCLq and the degree of polymerization of the polymer synthesized as exposed in the latter chapter.

The DP $(p)$ was calculated as described in eq. (S8):

$p=\frac{n M P C * G}{q * E}$

The conversion $X_{H E M A-Q C L q / M P C}$, was calculated according to eq. (S9):

$X_{H E M A Q C L q / M P C}=1-\frac{2 * q * A}{G}$ 


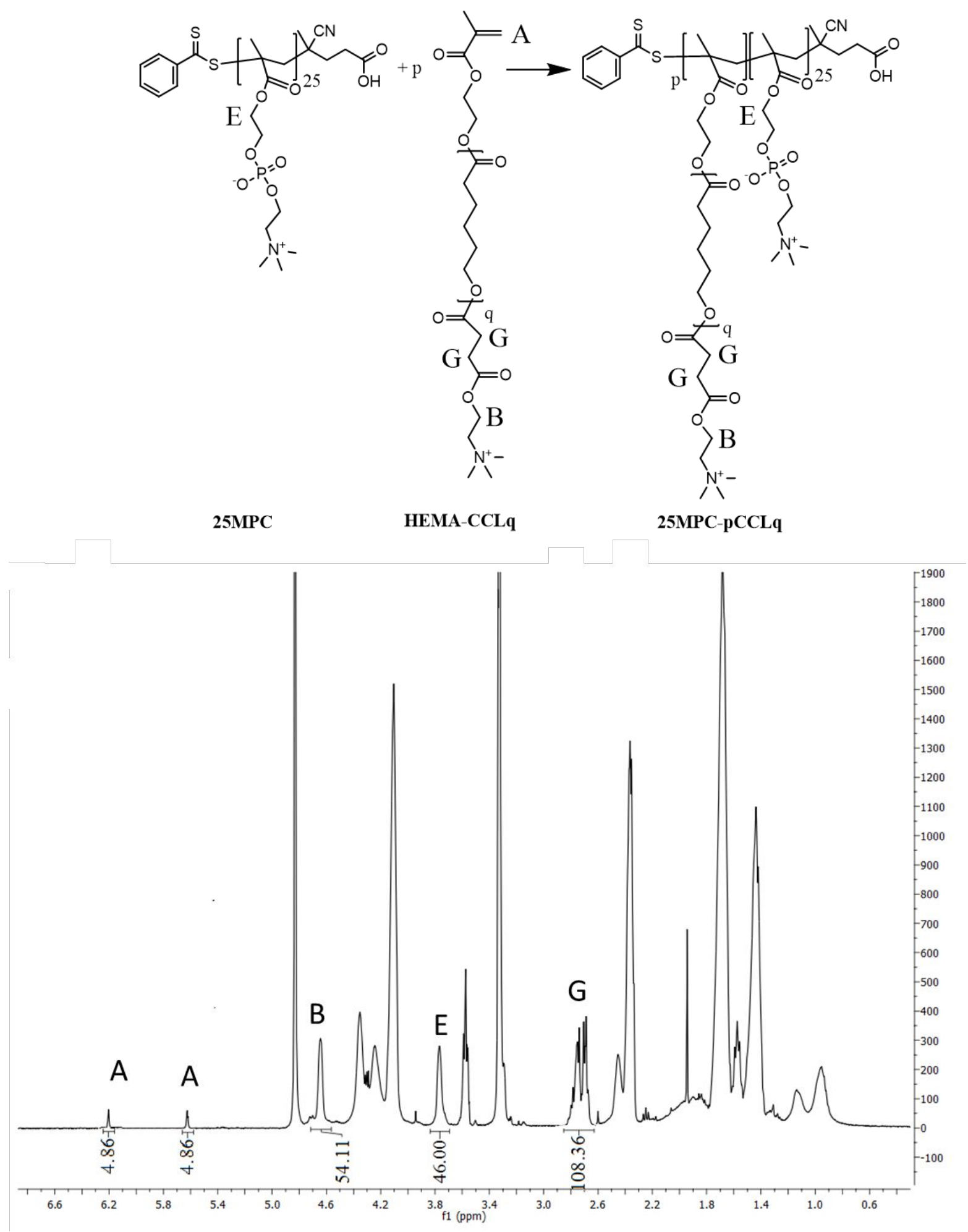

Figure S7: Representative ${ }^{1} \mathrm{H}-\mathrm{NMR}$ spectrum of the 25MPC-pCCLq-based nanoparticles with $\mathrm{q}=5$ and $\mathrm{p}=27$ and conversion evaluation 
Analyzing this spectrum, it was possible to calculate the conversion with respect to the macromonomer HEMA-CCLq and the degree of polymerization of the polymer synthesized as exposed in the latter chapter.

The $\operatorname{DP}(p)$ was calculated as described in eq. (S10):

$p=\frac{n M P C * G}{2 E}$

The conversion $X_{H E M A-Q C L q / M P C}$, was calculated according to eq. (S11):

$X_{H E M A C C L q / M P C}=1-\frac{2 A}{G}$

Table S1. Characterization of the macromonomer and of the zwitterionic macro-RAFT agent

\begin{tabular}{cccccc}
\hline Sample & $\begin{array}{c}\mathrm{Mn}_{\mathrm{GPC}} \\
{[\mathrm{g} / \mathrm{mol}]}\end{array}$ & $\begin{array}{c}Ð_{\mathrm{GPC}} \\
{[-]}\end{array}$ & $\begin{array}{c}q_{\mathrm{NMR}} \\
{[-]}\end{array}$ & $\begin{array}{c}n_{\mathrm{NMR}} \\
{[-]}\end{array}$ & $\begin{array}{c}X \\
{[\%]}\end{array}$ \\
\hline HEMA-CL $_{3}$ & $451^{*}$ & 1.33 & 3.21 & - & 98 \\
HEMA-CL $_{5}$ & 754 & 1.29 & 5.17 & - & 98 \\
\hline HEMA-QCL $_{3}$ & 602 & 1.36 & 3.21 & - & $>99$ \\
HEMA-QCL $_{5}$ & 814 & 1.37 & 5.17 & - & 94 \\
\hline HEMA-CCL $_{3}$ & 680 & 1.29 & 3.21 & - & 83 \\
HEMA-CCL & 910 & 1.31 & 5.17 & - & 85 \\
\hline 25MPC & 4794 & 1.16 & - & 23.12 & 99 \\
\hline
\end{tabular}

*Extrapolated from the calibration curve 
Table S2. Nanoparticle Characterization: degree of polymerization as determined via NMR for the macromonomer (qNMR) and the hydrophobic block (pNMR), monomer conversion, volume average diameter (Dv), Z-average, polydispersity index (PDI) and surface $\zeta$-potential $(\zeta)$.

\begin{tabular}{|c|c|c|c|c|c|c|c|}
\hline $\begin{array}{c}\text { Sample } \\
-\end{array}$ & $\begin{array}{c}\mathrm{q}_{\mathrm{NMR}} \\
{[-]}\end{array}$ & $\begin{array}{c}\mathrm{p}_{\mathrm{NMR}} \\
{[-]}\end{array}$ & $\begin{array}{c}X \\
{[\%]}\end{array}$ & $\begin{array}{c}D v \\
{[\mathrm{~nm}]}\end{array}$ & $\begin{array}{c}\text { Z-average } \\
{[\mathrm{nm}]}\end{array}$ & $\begin{array}{l}P d I \\
{[-]}\end{array}$ & $\begin{array}{c}\zeta \\
{[-]}\end{array}$ \\
\hline 25MPC-15CL3 & 3.21 & 19 & $97 \%$ & $15.3 \pm 0.1$ & $18.6 \pm 0.1$ & $0.13 \pm 0.02$ & $-11.0 \pm 6.5$ \\
\hline 25MPC-30CL3 & 3.21 & 36 & $99 \%$ & $32.5 \pm 0.2$ & $43.1 \pm 0.2$ & $0.186 \pm 0.07$ & $-13.9 \pm 3.2$ \\
\hline 25MPC-60CL3 & 3.21 & 60 & $99 \%$ & $69.0 \pm 0.2$ & $79.1 \pm 0.2$ & $0.09 \pm 0.06$ & $-20.5 \pm 1.9$ \\
\hline 25MPC-15CL5 & 5.17 & 19 & $96 \%$ & $23.4 \pm 0.2$ & $31.6 \pm 0.2$ & $0.18 \pm 0.01$ & $-18.1 \pm 1.2$ \\
\hline 25MPC-30CL5 & 5.17 & 33 & $98 \%$ & $51.4 \pm 0.1$ & $58.9 \pm 0.1$ & $0.09 \pm 0.07$ & $-20.0 \pm 5.6$ \\
\hline 25MPC-60CL5 & 5.17 & 67 & $99 \%$ & $109.9 \pm 0.7$ & $115.4 \pm 0.7$ & $0.06 \pm 0.07$ & $-22.6 \pm 2.8$ \\
\hline 25MPC-15QCL3 & 3.21 & 16 & $97 \%$ & $11.6 \pm 0.5$ & $15.4 \pm 0.5$ & $0.23 \pm 0.01$ & $-17.9 \pm 6.8$ \\
\hline 25MPC-30QCL3 & 3.21 & 31 & $98 \%$ & $28.7 \pm 0.7$ & $42.8 \pm 0.7$ & $0.18 \pm 0.06$ & $-25.2 \pm 2.5$ \\
\hline 25MPC-60QCL3 & 3.21 & 57 & $96 \%$ & $57.3 \pm 0.4$ & $76.9 \pm 0.4$ & $0.10 \pm 0.04$ & $-43.9 \pm 10.5$ \\
\hline 25MPC-15QCL5 & 5.17 & 20 & $95 \%$ & $35.5 \pm 0.1$ & $45.9 \pm 0.1$ & $0.19 \pm 0.003$ & $-12.3 \pm 11.7$ \\
\hline 25MPC-30QCL5 & 5.17 & 30.7 & $89 \%$ & $47.3 \pm 0.1$ & $58.1 \pm 0.1$ & $0.12 \pm 0.03$ & $-35.2 \pm 0.6$ \\
\hline 25MPC-60QCL5 & 5.17 & 57.1 & $89.8 \%$ & $114.1 \pm 0.8$ & $121.0 \pm 0.8$ & $0.10 \pm 0.02$ & $-62.3 \pm 2.5$ \\
\hline 25MPC-15CCL3 & 3.21 & 13 & $89 \%$ & $29.9 \pm 1.6$ & $33.1 \pm 0.3$ & $0.19 \pm 0.01$ & $+9.2 \pm 1.4$ \\
\hline 25MPC-30CCL3 & 3.21 & 34 & $94 \%$ & $40.5 \pm 2.4$ & $51.4 \pm 0.1$ & $0.17 \pm 0.01$ & $+9.0 \pm 1.4$ \\
\hline 25MPC-60CCL3 & 3.21 & 55 & $91 \%$ & $65.3 \pm 8.4$ & $78.6 \pm 0.4$ & $0.18 \pm 0.01$ & $+14.2 \pm 5.1$ \\
\hline 25MPC-15CCL5 & 5.17 & 16 & $96 \%$ & $24.2 \pm 0.5$ & $31.9 \pm 0.5$ & $0.23 \pm 0.03$ & $+5.4 \pm 2.6$ \\
\hline 25MPC-30CCL5 & 5.17 & 27 & $91 \%$ & $105.8 \pm 1.3$ & $118.1 \pm 0.5$ & $0.19 \pm 0.01$ & $+27.5 \pm 8.7$ \\
\hline 25MPC-60CCL5 & 5.17 & 54 & $88 \%$ & $127.6 \pm 1.6$ & $134.2 \pm 0.7$ & $0.20 \pm 0.01$ & $+15.5 \pm 7.4$ \\
\hline
\end{tabular}



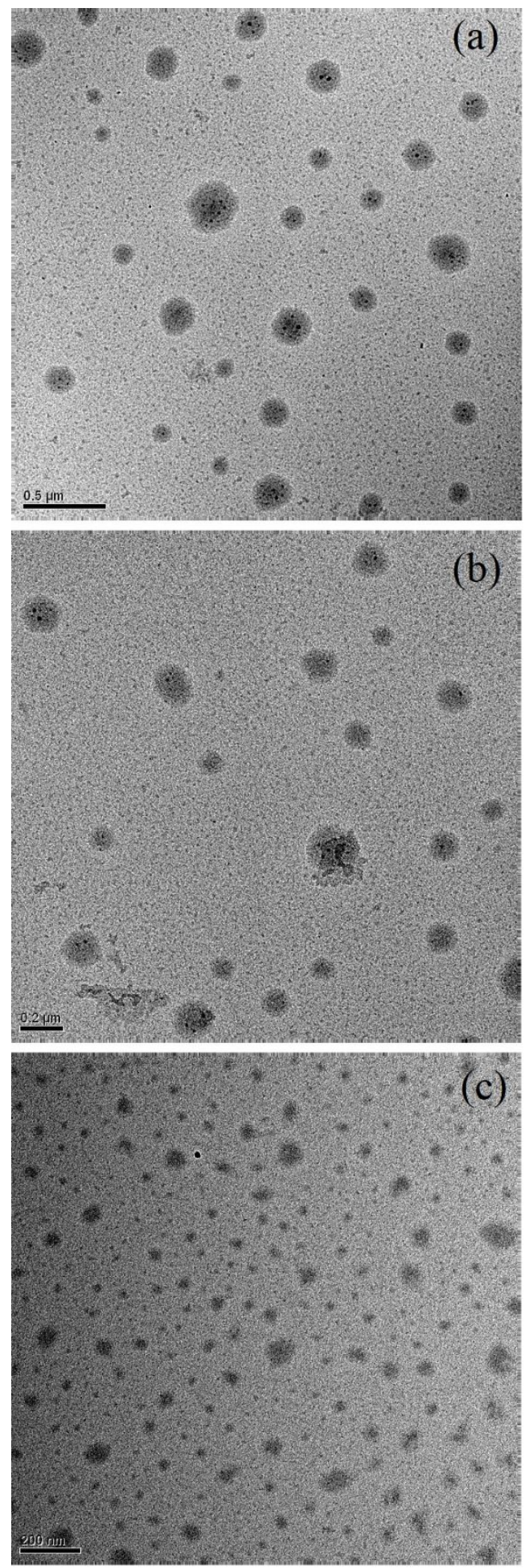

Figure S8: TEM micrographs of: (a) 25MPC-30CL5, (b) 25MPC-30QCL5, and (c) 25MPC-30CCL5. 


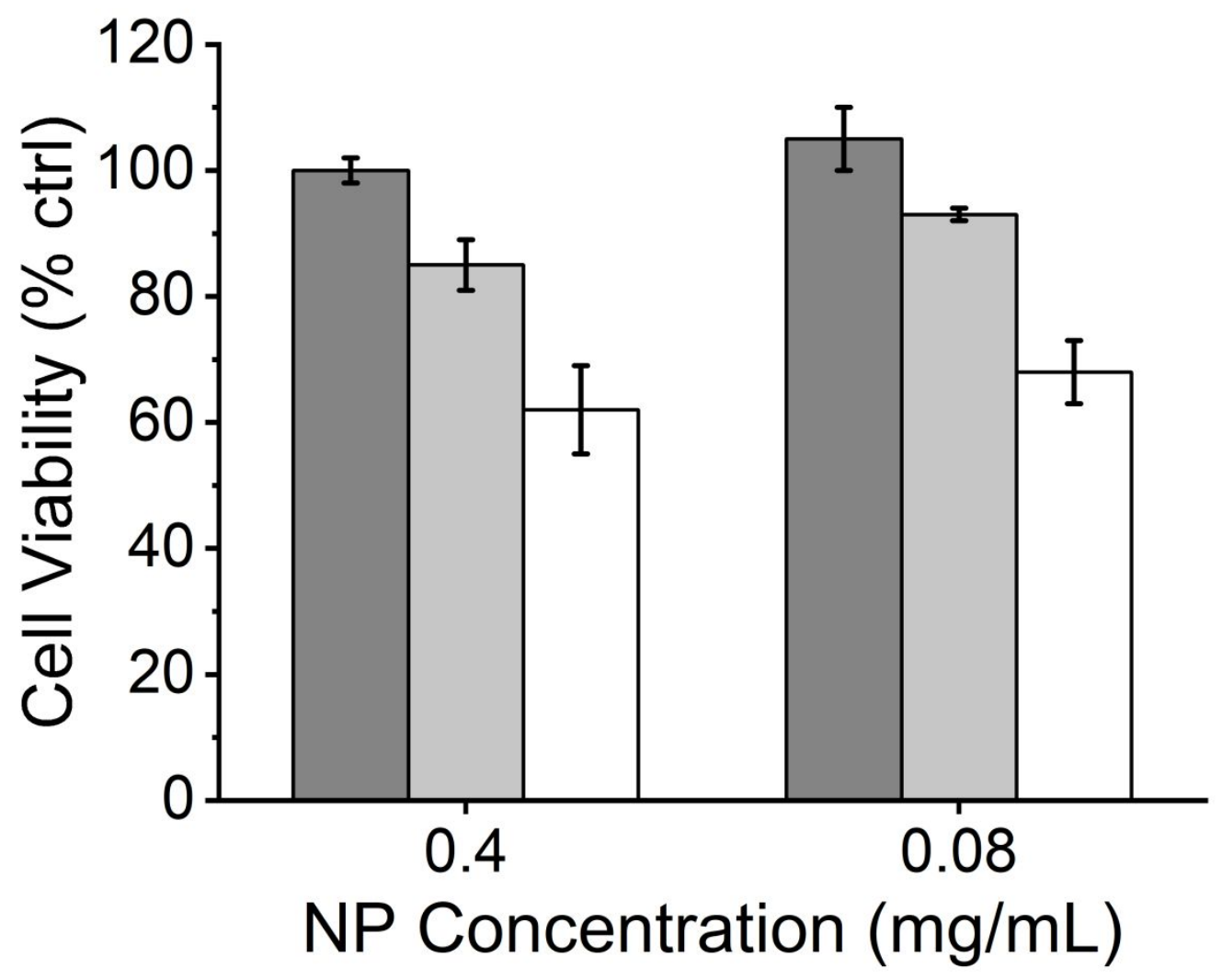

Figure S9: Cell viability, expressed as the percentage of cells with respect to the control, after $72 \mathrm{~h}$ incubation with 25MPC-30CL5 (dark grey bars), 25MPC-30QCL5 (light grey bars) and 25MPC-30CCL5 (white bars) NPs

Table S3. Dv and PdI of the nanoparticles before and after the freeze-drying step

\begin{tabular}{ccccc}
\hline Sample name & \multicolumn{2}{c}{ Before freeze-drying } & \multicolumn{2}{c}{ After freeze-drying } \\
{$[-]$} & Dv [nm] & PdI [-] & Dv [nm] & PdI [-] \\
\hline 25MPC-30CL5 & 60.52 & 0.061 & 82.94 & 0.044 \\
25MPC-30QCL5 & 87.24 & 0.077 & 103.7 & 0.156 \\
25MPC-30CCL5 & 117.25 & 0.136 & 114 & 0.162 \\
\hline
\end{tabular}

